\title{
- Electrospray Ionization and Low Energy Tandem Mass Spectrometry of Polyhydroxy Unsaturated Fatty Acids
}

\author{
Pat Wheelan, Joseph A. Zirrolli, and Robert C. Murphy \\ National Jewish Center for Immunology and Respiratory Medicine, Denver, Colorado, USA
}

\begin{abstract}
Negative ion electrospray ionization, fast-atom bombardment, and low energy tandem mass spectrometry were used for the analysis of dihydroxy-eicosatrienoic acids, which contain a vicinol diol and three nonconjugated double bonds, dihydroxy-eicosatetraenoic acids, which contain a conjugated triene structure, and trihydroxy-eicosatetraenoic acids, which contain a vicinol diol and a conjugated tetraene structure. In general, the product ion spectra were qualitatively similar for both modes of ionization, but electrospray ionization was strikingly more efficient in generation of abundant carboxylate anions that could be collisionally activated to yield product ion spectra. Collision-induced dissociation fragmentation mechanisms were described generally by $\alpha$-hydroxy fragmentations directed by relative positions of double bonds and were consistent with stable isotope labeling studies. Rearrangement of the conjugated triene system in dihydroxy-eicosatetraenoic acids may be described by formation of a cyclohexadiene structure. Fragmentations that involve a two-proton transfer were described best by intramolecular oxidation of a hydroxy substituent to an enolate that resulted in an extended conjugated system. Collision-induced dissociation spectra obtained for the polyhydroxy unsaturated fatty acids, which are isobaric within each class, were uniquely descriptive of individual structures. (J Am Soc Mass Spectrom 1996, 7, 140-149)
\end{abstract}

$\mathrm{O}$ xidation of arachidonic acid by enzymatic processes that involve cyclooxygenase or lipoxygenase activities or by nonenzymatic free radical processes results in the formation of hydroxy-substituted unsaturated fatty acids. Understanding these metabolic events and the factors that control metabolite formation requires unequivocable product identification because many of these metabolites are isobaric. Structure elucidation has relied in the past on gas chromatography-mass spectrometry analysis, which requires derivatization to volatile compounds [1-4]. More recently, high energy $[5,6]$ and low energy fast-atom bombardment (FAB) tandem mass spectrometry [7] of underivatized metabolites has become possible. However, analyses of mixtures of isomeric hydroxy-substituted unsaturated fatty acids found in biological samples usually necessitate prior purification, which normally is achieved by reverse phase high-performance liquid chromatography (HPLC) separation. The advent of electrospray ionization (ESI), which is compatible with high flow HPLC introduction of samples, suggested that ESI tandem mass spectrometry may be an attractive alternative for structural characterization of such arachidonic acid metabolites.

Address correspondence to Dr. Robert C. Murphy, National Jewish Center for Immunology and Respiratory Medicine, K929, 1400 Jackson Street, Denver, CO 80206.
Previous work that employed negative ion FAB tandem mass spectrometry for analysis of monohydroxy unsaturated fatty acids revealed collision-induced dissociation (CID) fragmentations that were dependent on both the position of hydroxy substitution and the positions of double bonds [7]. In that study, the CID of the carboxylate anions of monohydroxy unsaturated fatty acids was described primarily by five basic mechanisms (Scheme I). Type 1 involved an $\mathrm{O}$-hydro-C-allyl elimination charge-remote fragmentation at an allylic position that involved transfer of the hydroxy proton [8], whereas Type 2 described a charge-remote fragmentation at a vinylic position with prior rearrangement of the double bonds. Type 3 involved a charge-driven fragmentation at an allylic position and Type 4 involved a charge-driven fragmentation at a vinylic position with prior double bond rearrangement. Neither Type 3 nor Type 4 involved a hydrogen transfer. Type 5 described formation of a radical anion by homolytic fragmentation that involved an oxy-Cope rearrangement. This mechanism required the 3-hydroxy-1,5-hexadiene structure.

The present work extends this analysis to include polyhydroxy-substituted eicosanoids that have been analyzed by both FAB and ESI low energy tandem mass spectrometry. For all compounds studied, the tandem mass spectra obtained following ESI were identical to those obtained following FAB ionization, which suggests common fragmentation mechanisms 


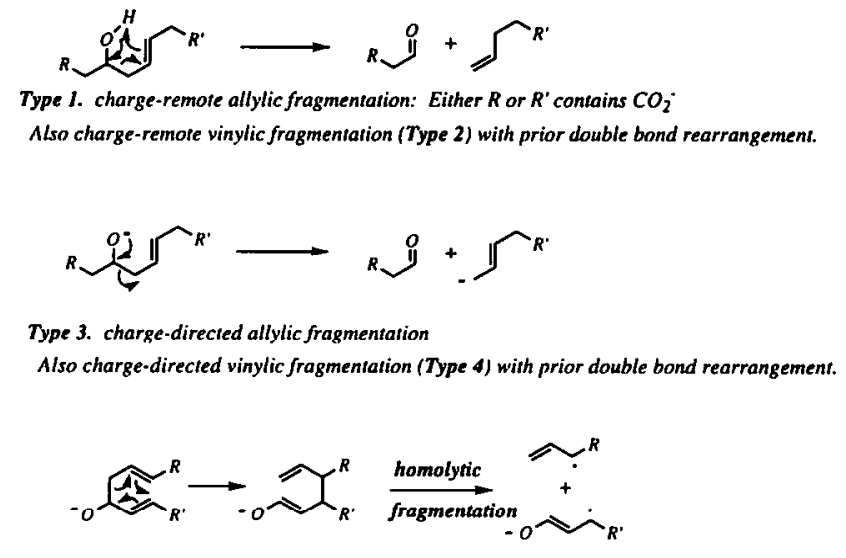

Type 5. oxy-Cope rearrangement and homolytic bond fragmentation

Scheme I. CID fragmentation mechanisms for hydroxy-substituted unsaturated fatty acids.

for both of these low energy processes. Mechanisms are proposed for the formation of fragment ions consistent with stable isotope labeling. Decomposition mechanisms appear to follow those proposed earlier for the monohydroxy fatty acids, but the presence of additional hydroxy groups and extended conjugation increased the complexity of the decomposition processes.

\section{Experimental}

All fatty acids were obtained from Cayman Chemical (Ann Arbor, MI). All other reagents were obtained from commercial sources at the highest grade possible.

For analysis by negative ion electrospray mass spectrometry (ESI-MS), compounds were evaporated to dryness under $\mathrm{N}_{2}$ and reconstituted in methanol:water (1:1) for a final concentration of $1-4 \mathrm{ng} / \mu \mathrm{L}$. Samples (1-5 $\mu \mathrm{L}$ ) were introduced into a Sciex Api III ${ }^{+}$triple quadrupole mass spectrometer (Perkin Elmer, Montreal, Canada) by flow injection through a $0.5-\mathrm{m} \times 50$ $\mu \mathrm{m}$ fused silica capillary with methanol:water (1:1) as the mobile phase, or MEOD: $\mathrm{D}_{2} \mathrm{O}(1: 1)$ for the deuterated samples, at a flow rate of $10 \mu \mathrm{L} / \mathrm{min}$. Spectra were acquired at $3 \mathrm{~s} / \mathrm{scan}$ by using a spray voltage of $-2400 \mathrm{~V}$ and an orifice voltage of $-60 \mathrm{~V}$ with a collisional offset potential of $20 \mathrm{eV}$. CID spectra were obtained with a pressure of argon in the second quadrupole equivalent to $200 \times 10^{12}$ molecules $/ \mathrm{cm}^{2}$, which resulted in a $40-80 \%$ reduction in the $[\mathrm{M}-$ $\mathrm{H}(\mathrm{D})]^{-}$precursor ion for most of the compounds studied. Variation of the argon pressure, with the collision offset potential held constant, altered the abundance of product ions relative to precursor ions, but did not alter the relative fragmentation pattern. Deuterium exchange of labile hydroxy and carboxy protons, indicated by $d_{3}$-OD-diHETrE, $d_{3}$-OD-diHETE, and $d_{4}$-ODlipoxin, was performed by dissolving the sample in $100-\mu \mathrm{L}$ MeOD, evaporating under $\mathrm{N}_{2}$, and redissolving the sample in MEOD: $\mathrm{D}_{2} \mathrm{O}$ (1:1). Exchange of carboxylate oxygens with ${ }^{18} \mathrm{O}$ was performed by treat- ment of the dried samples with 50 units of porcine liver esterase in $100-\mu \mathrm{L} \mathrm{H}_{2}{ }^{18} \mathrm{O}$ for $2 \mathrm{~h}$ at $37^{\circ} \mathrm{C}$ [9]. Samples were acidified with formic acid and extracted twice with ethyl acetate. The combined ethyl acetate extracts were dried under $\mathrm{N}_{2}$ and dissolved in methanol:water (1:1).

Negative ion FAB CID mass spectra were obtained on a Finnigan-MAT TSQ 70 (San Jose, CA) triple quadrupole mass spectrometer. Samples (10-40 $\mathrm{ng}$ in $1-\mu \mathrm{L}$ ethanol) were introduced by continuous flow injection by using methanol: $\mathrm{H}_{2} \mathrm{O}$ :glycerol (40:10:1) as the matrix at a flow rate of $5 \mu \mathrm{L} / \mathrm{min}$. The fast-atom bombardment gun (IonTech, Teddington, UK) was operated at $1 \mathrm{~mA}$ with xenon accelerated to $6 \mathrm{kV}$. Argon was used as the collision gas at a pressure that gave a $50 \%$ reduction in the abundance of the precursor ion. The collision offset energy $\left(E_{\mathrm{lab}}\right)$ was $30 \mathrm{eV}$.

\section{Results}

The analysis of polyhydroxy unsaturated fatty acid metabolites of arachidonic acid by negative ion mass spectrometry included dihydroxy-eicosatrienoic acids (diHETrEs), which contain a vicinol diol and three nonconjugated double bonds, dihydroxy-eicosatetraenoic acids (diHETEs), which contain a conjugated triene structure, and lipoxins, which contain a vicinol diol and a conjugated tetraene structure. These compounds produced abundant carboxylate anions [M $\mathrm{H}]^{-}$following either FAB ionization or ESI, and low energy CID of the carboxylate anion from each compound resulted in identical fragment ions for the two ionization processes (data not shown). The CID mass spectra showed common nonspecific product ions formed by the losses of $\mathrm{H}_{2} \mathrm{O}$ and $\mathrm{CO}_{2}$ from the carboxylate anion as well as compound-specific fragmentations that were dependent on hydroxy substituent position in relation to the position of double bonds.

\section{Dihydroxy-Eicosatrienoic Acids}

Fragment ions formed following CID of the molecular anion $(\mathrm{m} / \mathrm{z}$ 337) produced by ESI of the di-HETrE compounds all showed nonspecific losses of $\mathrm{H}_{2} \mathrm{O}(\mathrm{m} / z$ $319)$ and $2 \mathrm{H}_{2} \mathrm{O}(\mathrm{m} / z$ 301) with additional losses of $\mathrm{CO}_{2}$ from each of these fragment ions $(\mathrm{m} / z 275$ and 257, respectively; Figure 1).

5,6-diHETrE (Figure 1A). Formation of the most abundant product ion from 5,6-diHETrE (5,6-dihydroxy8,11,14-eicosatrienoic acid) ( $m / z$ 145) was consistent with charge-remote fragmentation of the $C-6, C-7$ bond with a proton transfer to the C-9 position and charge localized at the carboxy terminus. The transferred proton likely originated from either the hydroxy group (Scheme IIA, Type 1) or from C-5 (Scheme IIB) and resulted in loss of the neutral alkene and formation of either the keto or enol carboxylate anion. Both compet- 
(a)

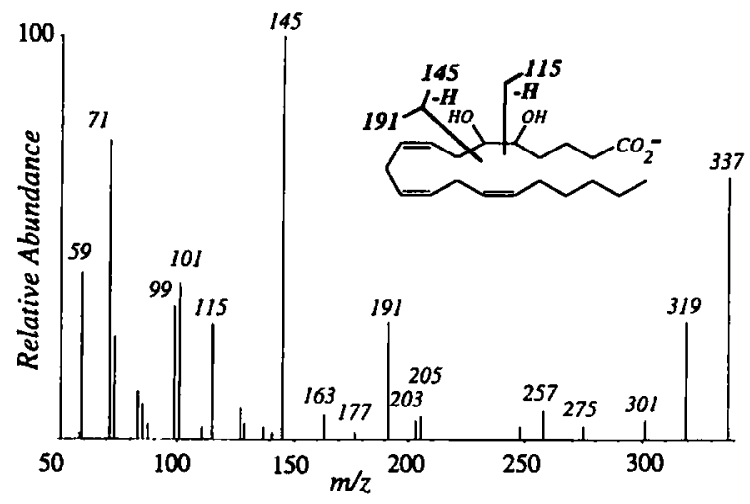

(c)

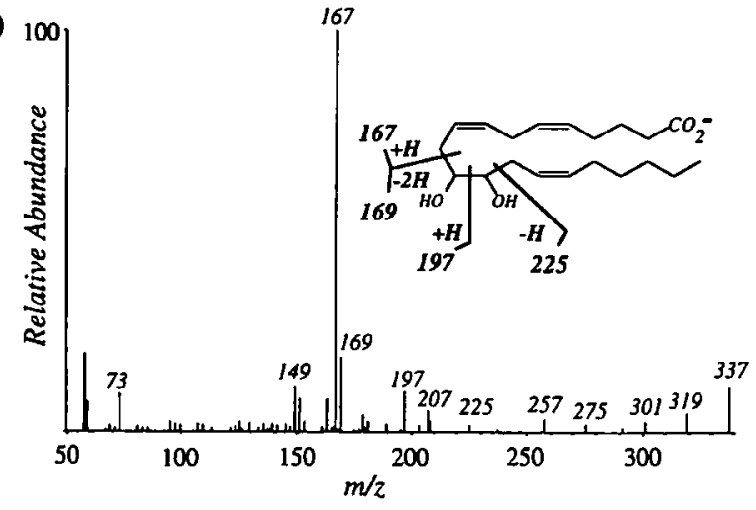

(b)

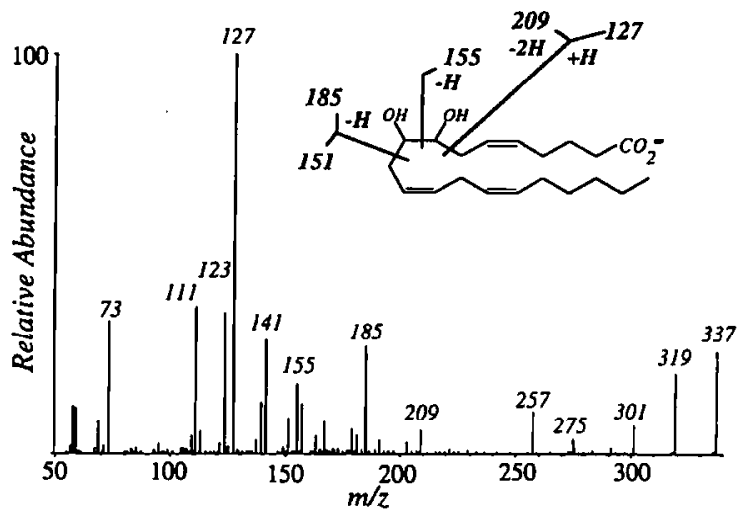

(d)

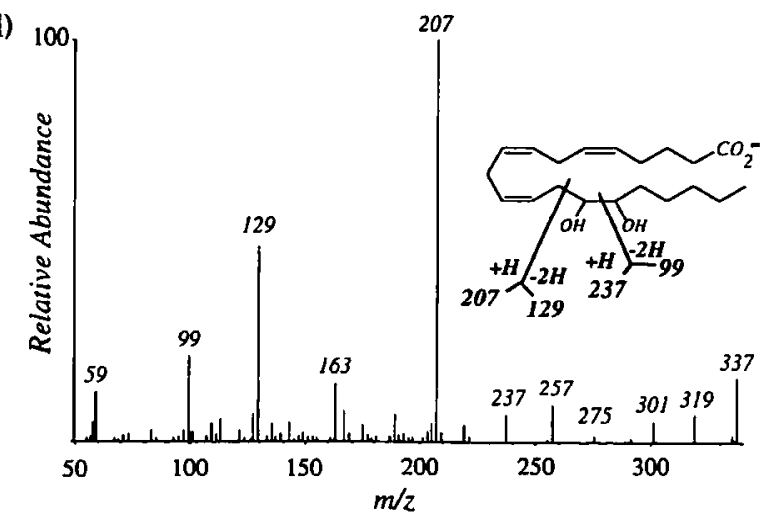

Figure 1. Negative ion product ion spectra of (A) 5,6-diHETrE $\left([\mathrm{M}-\mathrm{H}]^{-}, m / z\right.$ 337), (B) 8,9-diHETrE ([M - H $]^{-}, m / z$ 337), (C) 11,12-diHETrE ([M - H $]^{-}, m / z$ 337), and (D) 14,15-diHETrE $\left([\mathrm{M}-\mathrm{H}]^{-}, m / z 337\right)$.

ing mechanisms were supported by the CID spectrum of $d_{3}$-OD-5,6-diHETrE in which the ion at $\mathrm{m} / z 145$ was shifted to both $m / z 146(100 \%)$ and 147 (30\%) (Scheme IIA and B, respectively).

A less abundant ion observed at $\mathrm{m} / \mathrm{z} 191(30 \%)$ could arise following fragmentation of the C-6,C-7 bond, but with charge retained on the methyl terminus. This is consistent with a charge-driven fragmentation (Scheme III, Type 3) and does not retain a deuterium label in the CID spectrum of $d_{3}$-OD-5,6-diHETrE. A less abundant fragment ion observed at $m / z$ 192 (10\%) in the CID spectrum of $d_{3}$-OD-5,6diHETrE suggested competing mechanisms for this fragmentation as well. The second mechanism could involve the charge-remote fragmentation shown in

(a)

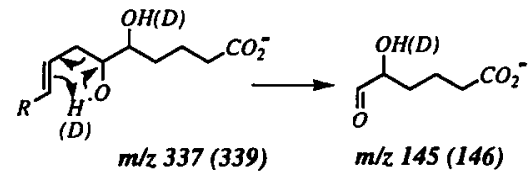

(b)

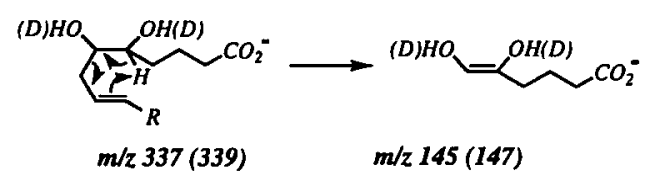

Scheme IIA with transfer of the hydroxy proton (or deuteron) to C-9, but with charge localized at one of the doubly allylic carbon positions, that is, at C-10 or $\mathrm{C}-13$. The product ion at $m / z 115(28 \%)$ could be due to a charge-remote fragmentation of the C-5,C-6 bond between the hydroxy substituents with loss of $\mathrm{H}_{2}$. Product ions observed at both $\mathrm{m} / \mathrm{z} 115(12 \%)$ and 116 (14\%) in the CID spectrum of $d_{3}$-OD-5,6-diHETrE suggested two competing mechanisms, both of which result in the formation of a C-5 aldehyde (Scheme IVA and B).

8,9-diHETrE (Figure 1B). 8,9-DiHETrE (8,9-dihydroxy$5,11,14$-eicosatrienoic acid) has the configuration in which each hydroxy-substituted carbon is two carbons removed from a double bond. Charge-remote fragmentation of the C-7,C-8 bond with proton (or deuteron) transfer to the C-5 position resulted in the terminal alkene carboxylate ion observed at $m / z 127$ (Scheme

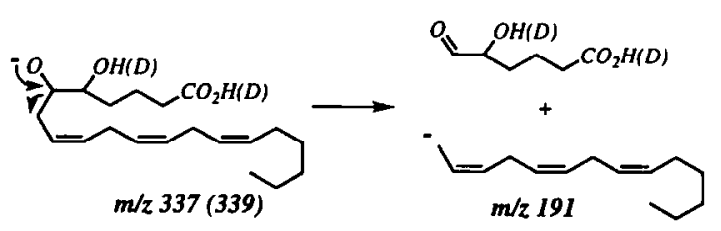

Scheme III 
(a)

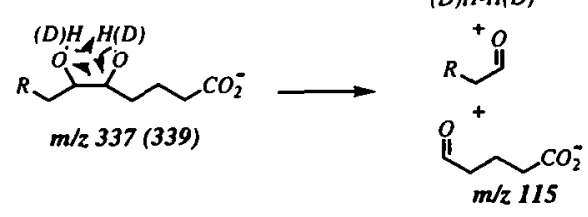

(b)

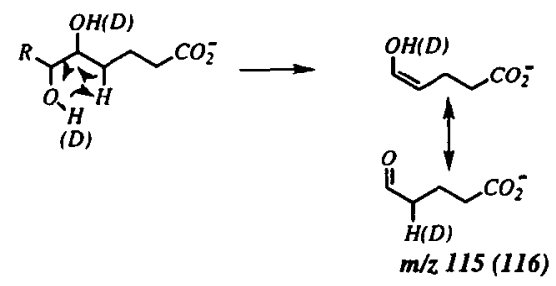

Scheme IV

VA, Type 1). Fragmentation of the C-9,C-10 bond with proton transfer to the $\mathrm{C}-12$ position resulted in the $\mathrm{C}-9$ aldehyde carboxylate ion at $m / z 185$ with the loss of a neutral terminal alkene (Scheme VB, Type 1). These mechanisms are supported by the CID spectrum of $d_{3}$-OD-8,9-diHETrE in which both ions, observed at $m / z 128$ and 186 , retained one deuterium. The ion observed at $m / z 141$ was consistent with loss of $\mathrm{CO}_{2}$ from $m / z 185$ and also retained one deuterium in the CID spectrum of $d_{3}$-OD-8,9-diHETrE. Loss of $\mathrm{H}_{2} \mathrm{O}$ from $\mathrm{m} / \mathrm{z} 141$ may result in the observed ion at $\mathrm{m} / \mathrm{z}$ 123 , but this ion did not involve loss of deuterium $(\mathrm{m} / \mathrm{z} 124$ in the deuterated compound), which suggests intermediate formation of a C-9 enolate structure and exchange of the hydroxy proton. The ion at $\mathrm{m} / \mathrm{z}$ 155 may be formed from the carboxylate anion by charge-remote fragmentation of the C-8,C-9 bond with loss of $\mathrm{H}_{2}$, as described for 5,6-diHETrE (Scheme IV) and was observed at $m / z 155(13 \%)$ and $156(7 \%)$ in the CID spectrum of $d_{3}$-OD-8,9-diHETrE. The observed ion at $m / z 111$ may be formed by loss of $\mathrm{CO}_{2}$ from $m / z 155$ and was observed at both $m / z 111$ $(20 \%)$ and $112(30 \%)$ in the deuterated analog.

A less abundant ion at $m / z 151$ was due to a charge-driven fragmentation (Type 2) of the C-9,C-10 bond, which resulted in formation of a stabilized anion with the loss of a neutral aldehyde analogous to that observed for 5,6-diHETrE (Scheme III). This ion was not shifted in the CID spectrum of the deuterated analog. Another ion that did not retain deuterium was observed at $m / z$ 209, which may be due to charge-remote fragmentation of the C-7,C-8 bond, but with the

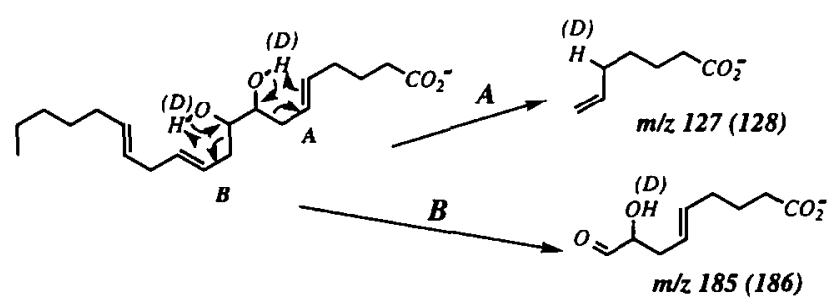

Scheme V

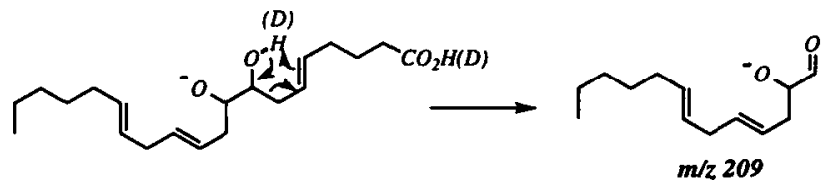

Scheme VI

charge site localized at the C-9 alkoxy position (Scheme VI).

11,12-diHETrE (Figure 1C). The configuration of 11,12-diHETrE (11,12-dihydroxy-5,8,14-eicosatrienoic acid) is analogous to the 8,9 isomer in that each hydroxy-substituted carbon is $\beta$ to a double bond. Charge-remote fragmentation (Type 1) of the C-10, $\mathrm{C}-11$ bond with proton transfer to the $\mathrm{C}-8$ position resulted in the terminal alkene carboxylate ion observed at $m / z$ 167. Charge-remote fragmentation (Type 1) of the $C-12, C-13$ bond with proton transfer to the C-15 position resulted in the C-11 aldehyde carboxylate ion at $\mathrm{m} / \mathrm{z} 225$ with the loss of a neutral terminal alkene. These pathways are analogous to those described for 8,9-diHETrE (Scheme V). These mechanisms are supported by the CID spectrum of $d_{3}$-OD11,12-diHETrE in which both ions, observed at $\mathrm{m} / \mathrm{z}$ 168 and $m / z 226$, retained one deuterium. Further loss of $\mathrm{H}_{2} \mathrm{O}$ from $m / z 225$ would result in the observed ion at $m / z$ 207. Surprisingly, this ion retained deuterium in the CID spectrum of $d_{3}$-OD-11,12-diHETrE, which suggests that the loss of $\mathrm{H}_{2} \mathrm{O}$ did not involve the $C-11$ hydroxy proton.

The ion observed at $m / z 169$, which did not shift in the CID spectrum of the deuterated analog, likely was formed by charge-remote fragmentation (Type 1) of the $C-10, C-11$ bond with the charge site localized as the C-12 alkoxide, analogous to formation of $m / z 209$ from 8,9-diHETrE (Scheme VI). Fragmentation between the hydroxy substituents also was observed for 11,12-diHETrE with formation of the ion at $\mathrm{m} / \mathrm{z} 197$. This ion shifted by two atomic mass units in the CID spectrum of $d_{3}$-OD-11,12-diHETrE, which suggests formation of a C-11 terminal hydroxy moiety by transfer of the C-12 hydroxy proton to $C-11$ and loss of a neutral aldehyde.

14,15-diHETrE (Figure 1D). Like the 5,6 isomer, 14,15-diHETrE (14,15-dihydroxy-5,8,11-eicosatrienoic acid) has the configuration that only one of the carbons containing a hydroxy substituent is in a position two carbons removed from a double bond. With charge localized as the carboxylate anion, charge-remote fragmentation (Type 1) of the C-13,C-14 bond with proton transfer to $\mathrm{C}-11$ resulted in the most abundant product ion at $m / z 207[\mathrm{~m} / z 208(100 \%)$ in the CID spectrum of $d_{3}-\mathrm{OH}-14,15$-diHETrE]. A less abundant ion at $\mathrm{m} / \mathrm{z}$ $209(50 \%)$ also was observed in the CID spectrum of $d_{3}$-OH-14,15-diHETrE, which may be due to the same charge-remote fragmentation with transfer of the C-14 
alkoxy deuterium to $\mathrm{C}-11$, but with the charge localized at one of the doubly allylic carbon positions, C-7 or C-10, rather than at the carboxy moiety that would retain the second deuterium. With charge localized as the C-15 alkoxide anion, a charge-remote fragmentation that involves the same $\mathrm{C}-13, \mathrm{C}-14$ bond resulted in an observed ion at $m / z 129$, analogous to that depicted in Scheme VI. As expected, this ion was not shifted in the spectrum of the deuterated analog.

\section{Dihydroxy-Eicosatetraenoic Acids}

Collisional activation of the dihydroxy-eicosatetraenoic carboxylate anions $(m / z 335)$ produced in ESI resulted in the loss of $\mathrm{H}_{2} \mathrm{O}\left(\mathrm{m} / z\right.$ 317) and further loss of $\mathrm{CO}_{2}$ $(m / z$ 273) for 5,6-diHETE, 5,12-diHETE, 5,15-diHETE, and for 8,15-diHETE (Figure 2). Additional fragmentations of the carbon skeleton were directed by the positions of hydroxy groups and double bonds and resulted in unique CID spectra for each isomer.

5,6-diHETE (Figure 2A). The major fragment ion produced under CID conditions from the carboxylate anion of 5,6-diHETE (5,6-dihydroxy-7,9,11,14-eicosatetraenoic acid) $m / z 115$, is formed by charge-remote fragmentation of the C-5,C-6 bond with transfer of the C-5 hydroxy proton to C-8 (Scheme VIIA). Consistent with this mechanism, this ion was unchanged in the CID spectrum of $d_{3}$-OD-5,6-diHETE. Chargedriven fragmentation of the same bond with charge retained on the methyl terminus (Type 3) resulted in a stabilized anion observed at $m / z 219$, which contained the C-6 hydroxy proton and was shifted to $m / z 220$ in the CID spectrum of $d_{3}$-OD-5,6-diHETE (Scheme VIIB).

$\alpha$-Hydroxy fragmentation of the $\mathrm{C}-6, \mathrm{C}-7$ bond occurred at a position vinylic to the double bond at C-7 and likely involved prior double bond rearrangement. Rearrangement of the conjugated triene to a cyclohexadiene structure followed by charge-driven fragmentation (Type 4) may result in the observed ion at $m / z$ 189 (Scheme VIIIA). This ion was not shifted in the CID spectrum of $d_{3}$-OD-5,6-diHETE. The ion observed at $m / z 163$ may result from loss of acetylene from $m / z 189$ and also was not shifted in the CID spectrum of the deuterated compound. A charge-remote fragmentation (Type 2) from the cyclohexadiene structure resulted in an observed ion at $m / z 145$ (Scheme VIIIB). This ion retained one deuterium in the CID spectrum of $d_{3}$-OD-5,6-diHETE.

A minor fragment ion was observed at $\mathrm{m} / \mathrm{z} 203$ and also is observed in the CID spectrum of 5-oxoeicosatetraenoic acid [10]. This ion could arise from initial dehydration that involves the C- 6 hydroxy substituent to form an enolate structure followed by rearrangement to a 5-oxo structure (Scheme IX). Fragmentation of the C-5,C-6 bond with loss of a neutral ketene (a)

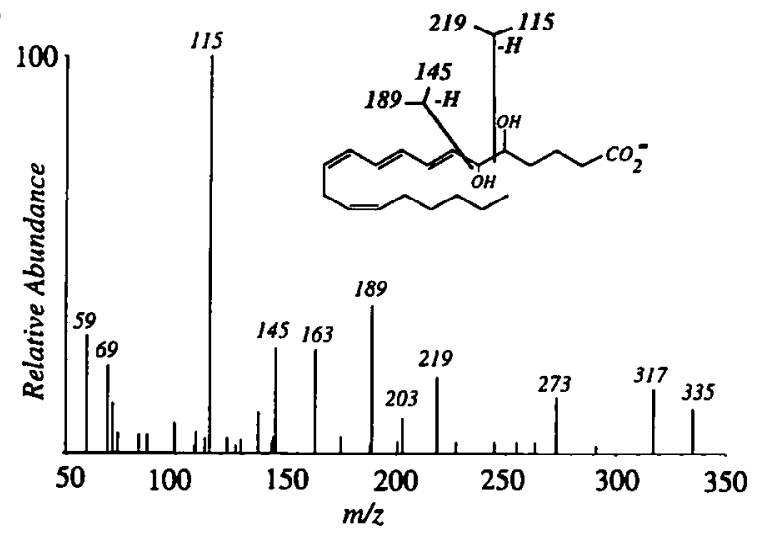

(c)

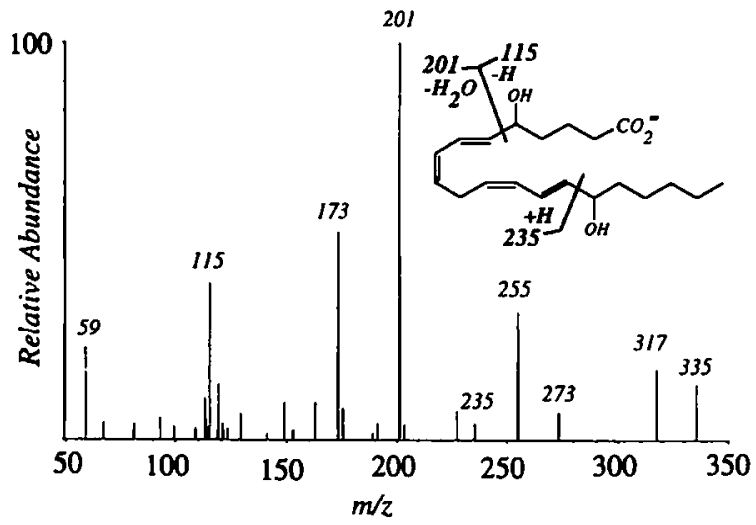

(b)

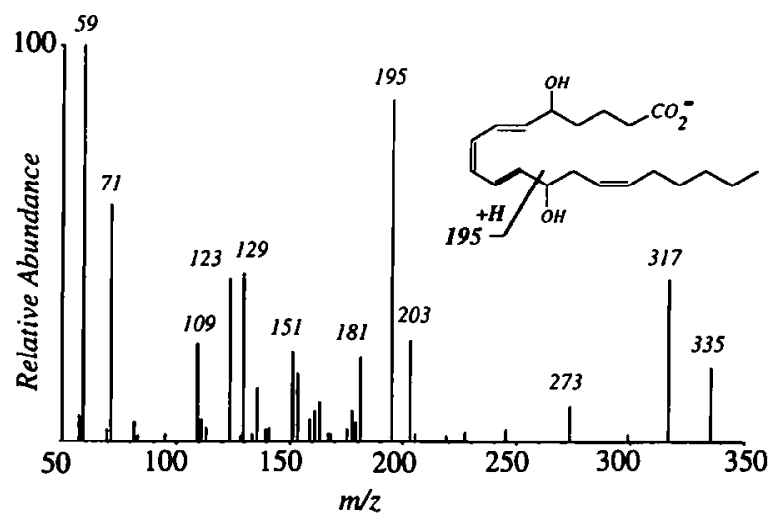

(d)

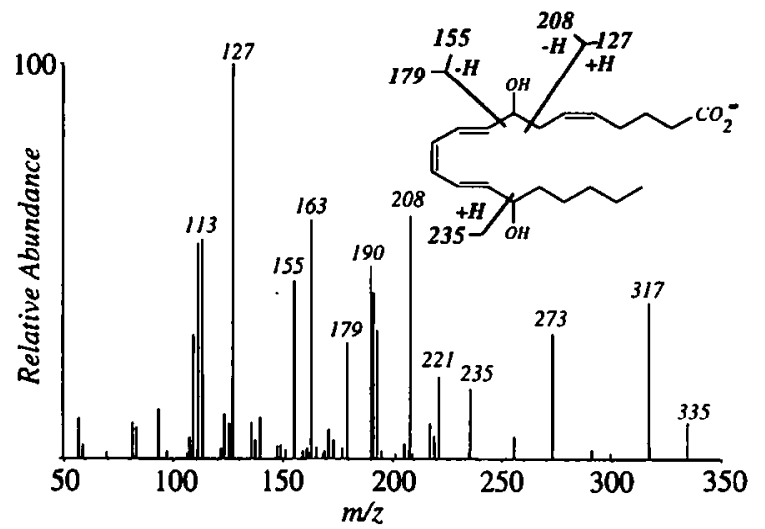

Figure 2. Negative ion product ion spectra of (A) 5,6-diHETE $\left([\mathrm{M}-\mathrm{H}]^{-}, m / z\right.$ 335), (B) 5,12-diHETE ([M $\left.-\mathrm{H}^{-}\right]^{-}, m / z$ 335), (C) 5,15-diHETE $\left([\mathrm{M}-\mathrm{H}]^{-}, m / z\right.$ 335), and (D) 8,15-diHETE $\left([\mathrm{M}-\mathrm{H}]^{-}, m / z 335\right)$. 


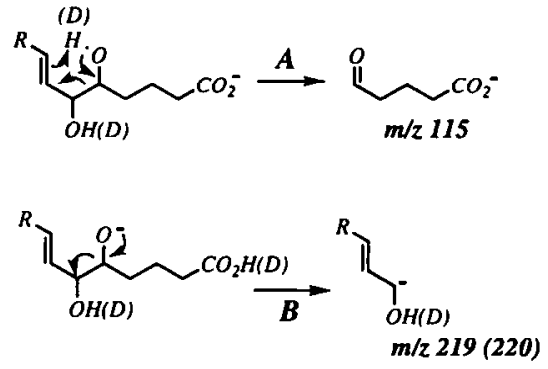

Scheme VII

would result in the observed ion that retained one deuterium in the deuterated analog.

5,12-diHETE (Figure 2B). The unique feature of 5,12diHETE $\quad(5,12$-dihydroxy-6,8,10,14-eicosatetraenoic acid) is the conjugated triene structure located between two hydroxy substituents. The most abundant product ion from 5,12-diHETE was observed at $m / z$ 195. This product ion shifted to $m / z 197$ in the product ion spectrum of $d_{3}$-OD-5,12-diHETE and was shifted to $m / z 199$ in the product ion spectrum of ${ }^{18} \mathrm{O}_{2}-5,12-$ diHETE. This was consistent with a charge-driven fragmentation with charge localized as the C-12 alkoxide ion and fragmentation of the $C-11, C-12$ bond. Fragmentation of this vinylic bond may occur with prior double bond rearrangement, which results in formation of a cyclohexadiene structure followed by C-5 proton abstraction by the C-12 alkoxide ion to form a C-5 enolate. Formation of the allylic stabilized ion at $m / z 195$ (Structure 1) would follow fragmentation and loss of a neutral aldehyde. Ions at $m / z 151$ and 123 correspond to losses of 44 and 72 , respectively, from $m / z$ 195, and may result from losses of $\mathrm{CO}_{2}$ and acrylic acid. These ions shifted to $m / z 153$ and 125 , respectively, in the CID spectrum of $d_{3}$-OD-5,12diHETE, but did not shift in the product ion spectrum of ${ }^{18} \mathrm{O}_{2}-5,12$-diHETE, which is consistent with loss of the carboxylate moiety.

Fragment ions were observed for 5,12-diHETE at $m / z 203$ and 129, which also were observed in the CID spectrum of 5-oxo eicosatetraenoic acid [10]. The mechanism for the formation of these ions may involve formation of the 5-oxo structure with dehydration that

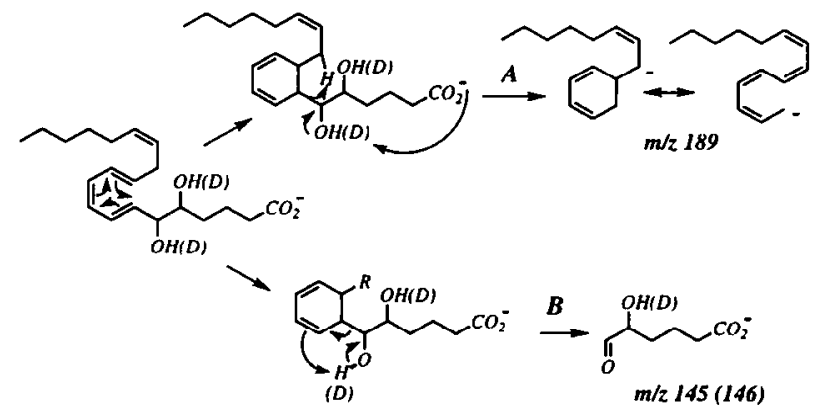

Scheme VIII

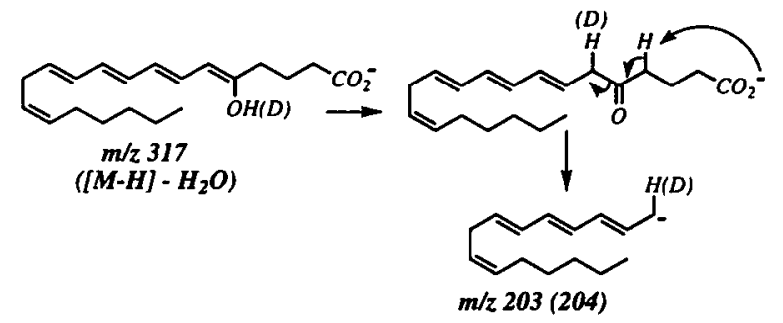

Scheme IX

involves the C-12 hydroxy substituent and the hydrogen at C-5. The ion at $m / z 203$ predominately shifted to $m / z \quad 204$ in the CID spectrum of $d_{3}$-OD-5,12diHETE and did not shift in the CID spectrum of ${ }^{18} \mathrm{O}_{2}-5,12$-diHETE. This ion may be formed by a mechanism analogous to that shown in Scheme IX. The ion at $m / z 129$ retained one deuterium in the CID spectrum of $d_{3}$-OD-5,12-diHETE and was shifted by $4 \mathrm{u}$ in the CID spectrum of ${ }^{18} \mathrm{O}_{2}-5,12$-diHETE (Structure 2). This ion may be formed from the $\mathrm{C}-5$ enolate structure by a mechanism analogous to the formation of $\mathrm{m} / \mathrm{z}$ 129 from 5-oxo-eicosatetraenoic acid [10].

5,15-diHETE (Figure 2C). The unique feature of 5,15diHETE $\quad(5,15$-dihydroxy-6,8,11,13-eicosatetraenoic acid) is the presence of the cross-conjugated diene moieties. The CID spectrum of 5,15-diHETE showed, in addition to the loss of one $\mathrm{H}_{2} \mathrm{O}$ moiety $(\mathrm{m} / z$ 317) and $\mathrm{H}_{2} \mathrm{O}$ and $\mathrm{CO}_{2}(\mathrm{~m} / z 273)$, a second prominent loss of $\mathrm{H}_{2} \mathrm{O}$ with an ion observed at $m / z 255$. This ion also was observed in the CID spectrum of $d_{3}$-OD-5,15diHETE, which reflected the loss of both OD substituents. A smaller contribution to this ion at $m / z 256$ (50\% of $\mathrm{m} / \mathrm{z} 255$ ) in the CID spectrum of $d_{3}$-OD-5,15diHETE may indicate the loss of water from a second minor pathway that does not involve the hydroxy proton.

The ions observed at $m / z 201$ and 115 were consistent with fragmentation of the C-5,C-6 bond. Fragmentation of this vinylic bond likely involved double bond rearrangement, possibly via a hydrogen transfer from C-10 to C-6 as proposed for 5-HETE [7]. The ion at $\mathrm{m} / \mathrm{z} 201$ then could be formed by a charge-driven fragmentation (Type 4) following dehydration that involved the C-15 hydroxy moiety (Scheme XA) and the ion at $m / z 115$ formed by a charge-remote fragmentation (Scheme XB, Type 2). Consistent with these mechanisms, neither ion retained deuterium in the CID spectrum of $d_{3}$-OD-5,15-diHETE.

The less abundant ion observed at $m / z 235(\mathrm{~m} / \mathrm{z}$ 237 for the $d_{3}$-OD-5,15-diHETE) would result from a charge-remote fragmentation of the $\mathrm{C}-14, \mathrm{C}-15$ bond

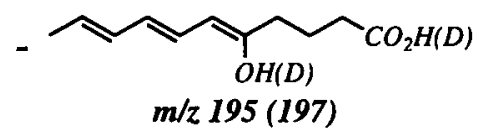

Structure 1 
<smiles>O=C([O-])CCCC(O)=CCO</smiles>

Structure 2

following double bond rearrangement (Type 2) of the $\mathrm{C}-11$ and $\mathrm{C}-13$ double bonds (Scheme $\mathrm{XI}$ ). Loss of $\mathrm{CO}_{2}$ and $\mathrm{H}_{2} \mathrm{O}$ (or HOD) from this ion would result in the ion observed at $m / z 173(\mathrm{~m} / z \quad 174$ for the deuterated analog).

8,15-diHETE (Figure 2D). The carbon skeleton of 8,15-diHETE (8,15-dihydroxy-5,9,11,13-eicosatetraenoic acid) was unique among the diHETEs studied in that it contains the 3-OH-1,5 diene structure (C-5 through C-10). This structural feature permitted an oxy-Cope type rearrangement (Type 5). Fragmentation by this mechanism produced the odd-electron ion at $\mathrm{m} / \mathrm{z} 208$ (Scheme XII) as an abundant product ion, and dehydration that involved the $\mathrm{C}-15$ hydroxy substituent from this ion yielded the product ion observed at $\mathrm{m} / \mathrm{z}$ 190. The odd-electron ions for $d_{3}$-OD-8,15-diHETE were observed at $\mathrm{m} / \mathrm{z} 209$ and 190 as expected and were unchanged in the product ion spectrum of ${ }^{18} \mathrm{O}_{2}-8,15$ diHETE.

The base peak ion at $m / z 127$ resulted from a charge-remote fragmentation (Type 1) of the C-7,C-8 bond as observed for 8,9-diHETrE (Scheme VA). This ion contained the proton from the C-8 hydroxy position and was observed at $\mathrm{m} / \mathrm{z} 128$ in the product ion spectrum of $d_{3}$-OD-8,15-diHETE. The ion that resulted from this fragmentation was observed at $\mathrm{m} / \mathrm{z} 131$ in the CID spectrum of ${ }^{18} \mathrm{O}_{2}-8,15$-diHETE, which is consistent with retention of the carboxylate moiety. Fragmentations of the C-8,C-9 bond and C-14,C-15 bond most likely result from prior rearrangement of the conjugated triene structure to a cyclohexadiene structure. Charge-remote fragmentation (Type 2) of the C$8, C-9$ bond then resulted in formation of the ion observed at $m / z 155$ (Scheme XIIIA), which shifted to $m / z 159$ in the CID spectrum of ${ }^{18} \mathrm{O}_{2}-8,15$-diHETE and was not changed in the CID spectrum of $d_{3}$-OD-8,15diHETE. Charge-driven fragmentation of the same bond (Type 4) resulted in the ion observed at $m / z$ 179,

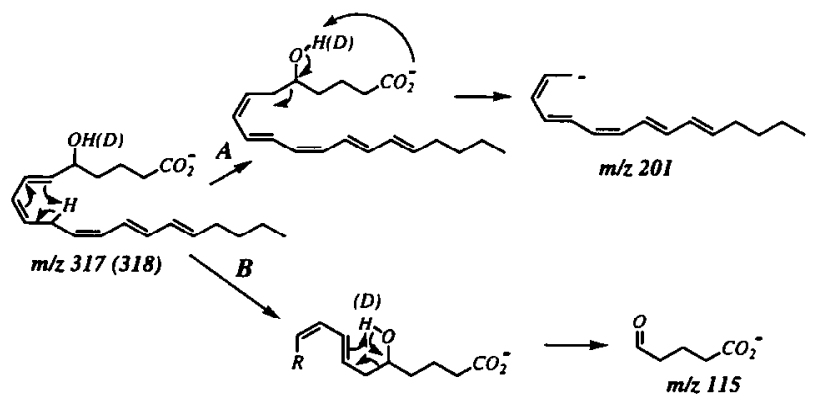

Scheme X

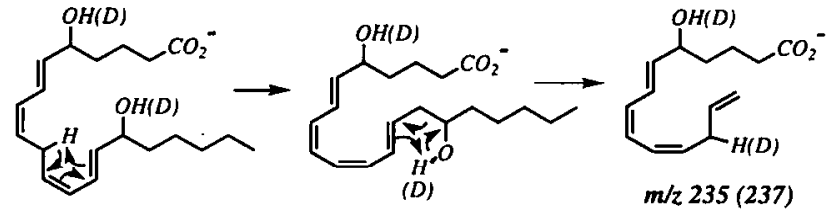

Scheme XI

which was observed at $m / z 180$ in the deuterated analog, but was unchanged in the CID spectrum of ${ }^{18} \mathrm{O}_{2}-8,15$-diHETE (Scheme XIIIB). The fragment ion at $m / z 235$ may be formed by a charge-driven fragmentation of the C-14,C-15 bond (Type 4) from the cyclohexadiene structure (Scheme XIIIC). This ion was shifted to $m / z 237$ in the CID spectrum of $d_{3}$-OD8,15-diHETE and was shifted to $m / z 239$ in the CID spectrum of ${ }^{18} \mathrm{O}_{2}-8,15$-diHETE. An abundant ion observed at $m / z 163$ may be formed by the mechanism proposed for the formation of the 5-oxo structure from 5,12-diHETE and is analogous to $m / z 203$ in 5,12diHETE. In the case of 8,15-diHETE this resulted in formation of a $\mathrm{C}-8$ enolate moiety. This ion was shifted to $m / z 164$ in the CID spectrum of $d_{3}$-OD-8,15diHETE and was unchanged in the CID spectrum of ${ }^{18} \mathrm{O}_{2}-8,15$-diHETE.

\section{Lipoxins}

Collisional activation of the carboxylate anions $(\mathrm{m} / \mathrm{z}$ 351) of lipoxin $\mathrm{A}_{4}$ and lipoxin $\mathrm{B}_{4}$ produced by ESI resulted in loss of $\mathrm{H}_{2} \mathrm{O}(\mathrm{m} / z 333)$, loss of $\mathrm{H}_{2} \mathrm{O}$ and $\mathrm{CO}_{2}(m / z 289)$, and loss of $2 \mathrm{H}_{2} \mathrm{O}$ and $\mathrm{CO}_{2}(\mathrm{~m} / z 271)$ (Figure 3 ).

Lipoxin $A_{4}$ (Figure $3 A$ ). The CID spectrum of the carboxylate anion of lipoxin $\mathrm{A}_{4}$ (5,6,15-trihydroxy7,9,11,13-eicosatetraenoic acid) was dominated by ions that resulted from fragmentation of the C-5,C-6 bond. Charge-remote fragmentation (Type 1) of this bond with transfer of the C-5 hydroxy proton to C-8 would yield the ion at $m / z 115$, analogous to that described for 5,6-diHETE (Scheme VIIA). Alternatively, fragmentation of this bond may occur with loss of $\mathrm{H}_{2}$ (both

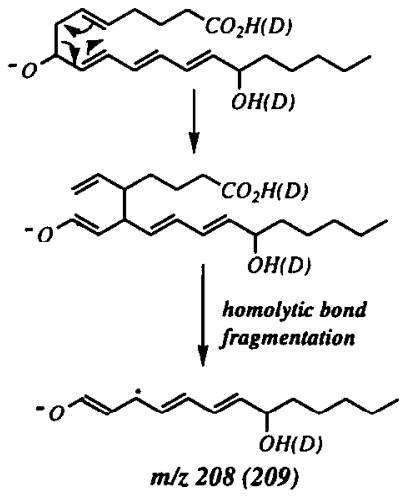

Scheme XII 

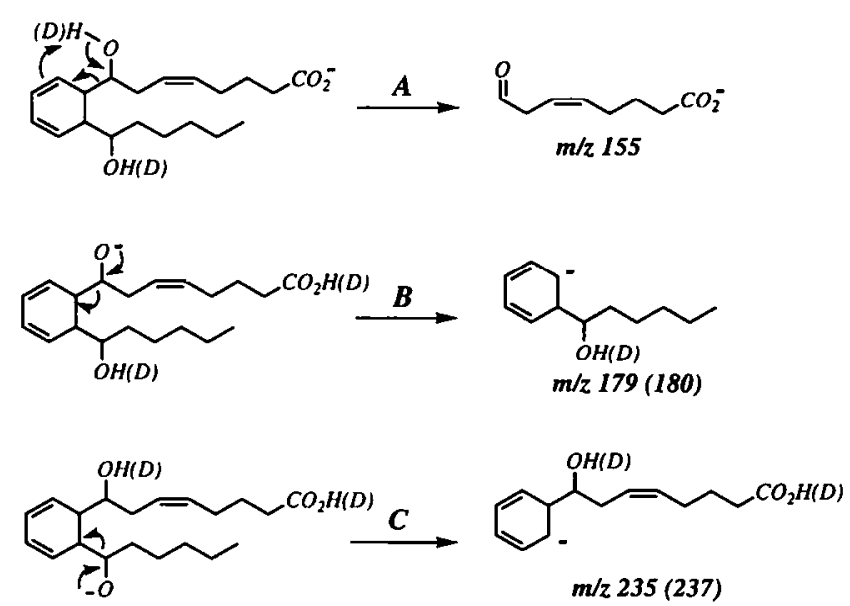

Scheme XIII

hydroxy protons) and formation of two terminal aldehydes as described for fragmentation between the diol in 5,6-diHETrE (Scheme IVA). This ion was unchanged in the spectrum of $d_{4}$-OD-lipoxin $\mathrm{A}_{4}$ and was shifted to $m / z 119$ in the CID spectrum of ${ }^{18} \mathrm{O}_{2}$-lipoxin $\mathrm{A}_{4}$, and the two mechanisms cannot be differentiated. With charge localized as the C-5 alkoxide, charge-driven fragmentation of the C-5,C-6 bond (Type 3) would result in the ion observed at $m / z 235$ with loss of 5-oxo-pentanoic acid, analogous to that described for 5,6-diHETE (Scheme VIIB). This ion was shifted to $m / z 237$ in the CID spectrum of $d_{4}$-OD-lipoxin $\mathrm{A}_{4}$, which reflects the presence of two - OD substituents. Loss of $\mathrm{H}_{2} \mathrm{O}$ from this ion produced the ion observed at $m / z 217$, which was shifted to $m / z 218$ in the CID spectrum of $d_{4}$-OD-lipoxin $\mathrm{A}_{4}$ consistent with the loss of HOD. Neither ion was shifted in the CID spectrum of ${ }^{18} \mathrm{O}_{2}$-lipoxin $\mathrm{A}_{4}$ as expected. The ion at $\mathrm{m} / \mathrm{z} 135$ may be due to loss of hexanal (C-15 through $\mathrm{C}-20$ ) from $m / z$ 235, which involves rearrangement of $C-9$ through $\mathrm{C}-14$ to a cyclohexadiene structure and trans-

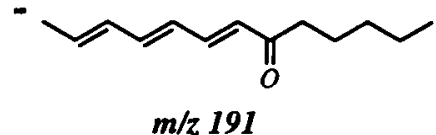

Structure 3

fer of the C-15 hydroxy proton with fragmentation of $\mathrm{C}-14, \mathrm{C}-15$ (Type 2). This ion was observed at $\mathrm{m} / z 137$ in the deuterated analog, which is consistent with retention of both C-6 and C-15 hydroxy protons, and was unchanged in the CID spectrum of ${ }^{18} \mathrm{O}_{2}$-lipoxin $\mathrm{A}_{4}$.

Lipoxin $B_{4}$ (Figure $3 B$ ). Decomposition of the carboxylate anion of lipoxin $B_{4}(5,14,15$-trihydroxy-6,8,10,12eicosatetraenoic acid) produced a much more complicated spectrum than was observed for lipoxin $\mathrm{A}_{4}$. Ions that resulted from fragmentation directed by the vicinal diol at C-14 and C-15 and that contained the carboxy terminus as evidenced by a shift of $4 \mathrm{u}$ in the CID spectrum of ${ }^{18} \mathrm{O}_{2}$-lipoxin $\mathrm{B}_{4}$ were observed at $\mathrm{m} / \mathrm{z}$ 221 and 233. Charge-remote fragmentation (Type 1) of $\mathrm{C}-14, \mathrm{C}-15$ that involved hydrogen transfer from either the C-15 hydroxy group or the C-16 proton to $\mathrm{C}-12$ resulted in the low abundance ion at $m / z 251$. The abundant ion at $m / z 233$ may be formed by subsequent dehydration of $\mathrm{m} / z 251$ or by the foregoing mechanism that involves the dehydrated anion $m / z$ 333. Alternatively, with charge localized as the C-15 alkoxide, charge-driven fragmentation (Type 3 ) of C$14, \mathrm{C}-15$ may occur without a hydrogen transfer to form the stabilized allyl anion and the neutral hexanal: Formation of $m / z 221$ may involve prior rearrangemnt of $\mathrm{C}-8, \mathrm{C}-13$ to a cyclohexadiene structure and then either charge-remote fragmentation (Type 2) of C-13,C-14 with a hydrogen transfer from either the $\mathrm{C}-14$ hydroxy group or the $\mathrm{C}-15$ proton or a charge-driven fragmentation (Type 4) of C-13,C-14 with the charge localized (a)

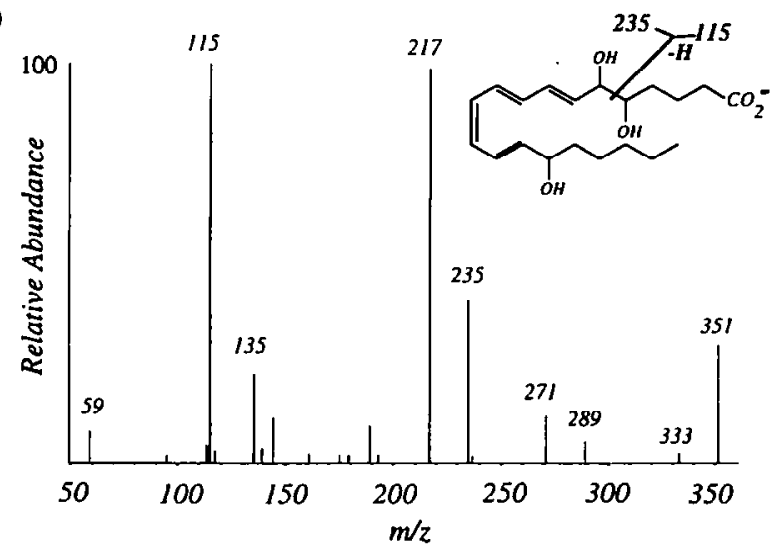

(b)

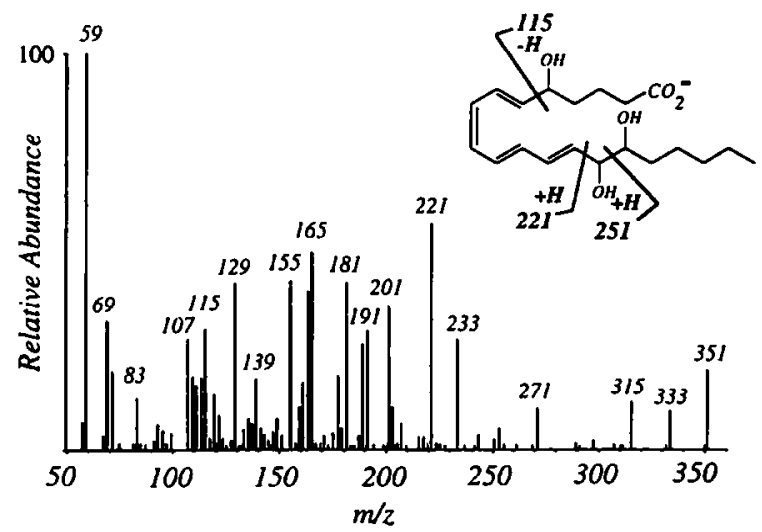

Figure 3. Negative ion product ion spectra of (A) lipoxin $A_{4}\left([M-H]^{-}, m / z 351\right)$ and (B) lipoxin $\mathrm{B}_{4}\left([\mathrm{M}-\mathrm{H}]^{-}, m / z 351\right)$. 
as the C-14 alkoxide. Evidence for operation of these multiple mechanisms was obtained from the CID spectrum of $d_{4}$-OD-lipoxin $\mathrm{B}_{4}$, which showed ions at both $m / z 223(30 \%)$ and $235(12 \%)$ as well as ions at $m / z$ $224(25 \%)$ and $236(11 \%)$. Fragmentation directed by the C-5 hydroxy substituent resulted in an observed ion at $m / z 115$, which contained the carboxy terminus as evidenced by an observed shift to $m / z 119$ in the CID spectrum of ${ }^{18} \mathrm{O}_{2}$-lipoxin $\mathrm{B}_{4}$. The mechanism for formation of $m / z 115$, which did not shift in the CID spectrum of $d_{4}$-OD-lipoxin $\mathrm{B}_{4}$, may be similar to the formation of this ion from 5,15-diHETE except with prior triene rearrangement to a cyclohexadiene structure.

A series of ions that differ by $26 \mathrm{u}$ and contain the carboxy terminus as evidenced by an increase of $4 \mathrm{u}$ in the CID spectrum of ${ }^{18} \mathrm{O}_{2}$-lipoxin $\mathrm{B}_{4}$ were observed at $m / z 129,155$, and 181 and involved apparent fragmentation of double bonds located in the conjugated tetraene structure. These ions may be formed by a mechanism similar to that for formation of $m / z 129$ from 5,12-diHETE (Structure 2) and would involve intermediate formation of a C-5 enolate structure. A second series of ions that differ by $26 \mathrm{u}$ and did not contain the carboxy terminus were observed at $m / z 191,165$, and 139. These ions were not shifted in the CID spectra of either $d_{4}$-OD-lipoxin $\mathrm{B}_{4}$ or ${ }^{18} \mathrm{O}_{2}$-lipoxin $\mathrm{B}_{4}$. A possible structure for the ion at $m / z$ 191, which resulted from fragmentation of the C-7,C-8 bond and involved dehydration of the C-14 hydroxy substituent, is shown in Structure 3. An additional ion that did not contain the carboxy terminus was observed at $m / z 201$. This ion was shifted to $m / z 202$ in the CID spectrum of $d_{4}$-OD-lipoxin $B_{4}$. Formation of this ion could involve loss of the hydroxy substituent at C-14 with the C-5 proton to form a 5-oxo structure analogous to that shown in Scheme IX. Fragmentation initiated by the carboxylate anion, which resulted in C-5,C-6 bond fragmentation following dehydration of the C-15 hydroxy substituent to form a C-15,C-16 double bond, would result in an ion structure for $\mathrm{m} / \mathrm{z} 201$ identical to that shown for 5,15-diHETE (Scheme X).

\section{Discussion}

In the negative ion mode, both FAB and ESI generated abundant carboxylate anions from polyhydroxy unsaturated fatty acids. The ESI method, which used 1-4 $\mathrm{ng} / \mu \mathrm{L}$, was significantly more sensitive than $\mathrm{FAB}$ analysis, where $10-40 \mathrm{ng} / \mu \mathrm{L}$ was required for comparable signal-to-noise ratio in the product ion spectra (data not shown). A similar increase in sensitivity has been observed in the ESI analysis of phospholipids as compared to FAB analysis [11]. Product ion spectra from the CID of carboxylate anions were qualitatively similar from both methods of ionization, however, and are summarized by class as follows:
DiHETrEs. The CID spectra of the carboxylate anions from the four isomeric diHETrEs studied are relatively simple due to the absence of any conjugated double bond systems and may be explained by two basic mechanisms: charge-remote fragmentation (Type 1) or charge-driven fragmentation (Type 3 ).

Several product ion series and ion abundance observations were discernible in these simple CID spectra. First, the most abundant product ion observed always corresponded to allylic fragmentation between the diol group and a double bond with charge retained with the carboxy terminus. Further, the tendency was to lose the diol group with the methyl terminus as the neutral hydroxy-aldehyde when possible. Thus, the most abundant ions observed in the CID spectra of $8,9-, 11,12-$, and 14,15-diHETrE were $m / z$ 127, 167, and 207 , respectively. This fragmentation was not possible with the 5,6-diol isomer because no double bond was present between the diol and the carboxylate group. The most abundant product ion observed from this species was an allylic fragmentation of the C-6,C-7 bond with retention of the aldehyde-hydroxy group with the carboxylate anion $m / z 145$.

Another trend observed in the CID of diHETrE carboxylate anions was that complementary ions also were observed with the most abundant ions produced, that is, fragmentation of the same bond but with charge on the methyl terminus. These ions were always of relatively low abundance and were observed at $m / z$ $191,209,169$, and 129 for 5,6-, 8,9-, 11,12-, and 14,15diHETrE, respectively.

DiHETEs. The diHETEs all contain $\alpha$-hydroxy conjugated polyene systems. Many of the product ions observed in the CID spectra of these isomers were explained by the basic five mechanisms proposed for the monohydroxy unsaturated fatty acids. Of particular significance were the apparent vinylic fragmentations between the hydroxy carbon and the conjugated double bonds. The mechanisms proposed for the monohydroxy unsaturated species involved prior rearrangement of the conjugated diene system through a 1,6 hydrogen shift to form a $\beta$-hydroxy conjugated diene and subsequent fragmentation of this bond [7]. Such a hydrogen rearrangement was not possible with the conjugated triene systems. Instead the apparent vinylic fragmentation observed in the diHETEs appeared to involve prior cyclization of the triene system to a cyclohexadienyl group in which the hydroxy-substituted carbon is two carbon atoms removed from the diene. Fragmentation of this rearranged structure often yields the most abundant product ions. This rearrangement was reasonable to propose because thermal cyclization of conjugated trienes has been shown during the gas chromatography-mass spectrometry analysis of diHETE derivatives $[4,12]$.

The clearest demonstration of these mechanisms was observed in the CID spectrum of the carboxylate anion of 8,15-diHETE (Figure 2D). As observed earlier 
[7], some ambiguity may exist in assigning particular fragmentations as either charge remote or charge driven. Thus, an abundant ion was produced from the 8,15-diHETE anion due to apparent vinylic fragmentation of $\mathrm{C}-14, \mathrm{C}-15, \mathrm{~m} / \mathrm{z}$ 235, which may be either a Type 2 or Type 4 fragmentation, whereas vinylic fragmentation of the C-8,C-9 bond $(m / z$ 155) must be Type 2 and vinylic fragmentation of the same bond that results in the observed ion at $m / z 179$ can be described only as a Type 4 fragmentation. Allylic fragmentation of the C-7,C-8 bond $(\mathrm{m} / z$ 127) may be either Type 1 or Type 3, and homolytic fragmentation following oxy-Cope rearrangement with loss of water, which resulted in an observed ion at $m / z$ 208, was described as Type 5 .

Of the diHETEs studied, the most complex CID spectrum was obtained from the carboxylate anion of 5,12-diHETE (Figure 2B). The most abundant product ion - $m / z \quad 195$ - is formed by apparent vinylic fragmentation of the C-11,C-12 bond, most likely following rearrangement of the triene group. Surprisingly, the analogous vinylic fragmentation of the C-5,C-6 bond was not observed ( $m / z 115)$ although the corresponding ion formation was observed in the other three isomeric diHETEs, that is, $m / z 115$ for 5,6 - and 5,15diHETE and $m / z 155$ for 8,15-diHETE. Instead, abundant product ions were observed that correspond to fragmentation between $\mathrm{C}-1$ and $\mathrm{C}-5$ at $\mathrm{m} / \mathrm{z} 71$ and 59 . Formation of these ions appeared to be facilitated for compounds that contain a C-5 hydroxy substituent, possibly a result of the proximity of the carboxylate anion. Further insight into the lack of C-5,C-6 fragmentation may be drawn from the formation of the abundant ions at $m / z 129$ and 203. These ions, which also were observed in the CID spectrum of 5-oxo-eicosatetraenoate [10], imply that an intramolecular oxidation of the C-5 hydroxy substituent that predominately affects fragmentation may occur. The CID spectrum of an isomeric compound, leukotriene $\mathrm{B}_{4}\left(\mathrm{LTB}_{4} ; 5,12\right.$-dihydroxy-6(Z),8( $E), 10(E), 14(Z)$-eicosatetraenoic acid), was identical to that of 5,12-diHETE. Fragmentation mechanisms for this compound and $\mathrm{LTB}_{4}$-derived metabolites are further discussed in the companion report [13].

Lipoxins. The lipoxins are similar in structure to the diHETEs in that they contain a polyhydroxy conjugated polyene system and CID mechanisms likely involve prior cyclization of the conjugated double bond system. The CID spectrum from the carboxylate anion of lipoxin $\mathrm{A}_{4}$ (Figure 3A) was analogous to that observed from 5,6-diHETE in that the 5,6-diol in both species predominately influenced fragmentation. The CID spectrum obtained from lipoxin $B_{4}$ (Figure 3B) also reflected the influence of the 14,15-diol group, but was more complex due to increased fragmentation of the unsaturated carbon chain, which also may involve prior intramolecular oxidation of either or both C-5 and C-14 hydroxy substituents.

In the previous work, collisional activation of the carboxylate anions from monohydroxy unsaturated fatty acids produced spectra that were both uniquely descriptive of individual species as well as predictive of isomeric and possible unknown species [7]. The five mechanisms proposed in that work described important CID processes that were competitive and yielded complex spectra. The predictive value of these mechanisms was based on observed trends in product ion formation that were dependent on specific positional relationships of the hydroxy group and unsaturated sites. In the present study the CID of carboxylate anions of polyhydroxy unsaturated fatty acids also are described mainly by these five competitive mechanisms, although the added functionalities of additional hydroxy substituents and extended conjugated unsaturation increased the complexity of the spectra. The extensive fragmentation and complexity diminished to some extent the predictive value of this technique for unknown species, but the resultant CID spectra were uniquely descriptive of each polyhydroxy structure.

\section{Acknowledgment}

This work was supported by a grant from the National Institutes of Health (HL25785).

\section{References}

1. Murphy, R. C.; Harper, T. W. In Mass Spectrometry in Biomedical Research; Gaskell, S. J., Ed.; Wiley: New York, 1986; PP 11-29.

2. Brezinski, D.; Serhan, C. N. Biol. Mass Spectrom. 1991, $20,45$.

3. Claeys, M. Mass Spectrom. Rev. 1989, 8, 1.

4. Wheelan, P.; Zirrolli, J. A.; Murphy, R. C. J. Am. Soc. Mass Spectrom. 1995, 6, 40.

5. Deterding, L. J.; Curtis, J. F.; Tomer, K. B. Biol. Mass Spectrom. 1992, 21, 597

6. Zhang, J. Y.; Nobes, B. J.; Wang, J.; Blair, I. A. Biol. Mass Spectrom. 1994, 23, 399.

7. Wheelan, P.; Zirrolli, J. A.; Murphy, R. C. Biol. Mass Spectrom. 1993, 22, 465.

8. Adams, J.; Gross, M. L. J. Am. Chem. Soc. 1989, 111, 435.

9. Westcott, J. Y.; Clay, K. L.; Murphy, R. C. Biomed. Mass Spectrom. 1985, 12, 714.

10. MacMillan, D. K.; Murphy, R. C. I. Am. Soc. Mass Spectrom. 1995, 6, 1190-1201.

11. Han, X.; Gross, R. W. Proc. Natl. Acad. Sci. USA 1994, 91 , 10635.

12. Borgeat, P.; Pilote, S. Prostaglandins 1988, 35, 723.

13. Wheelan, P.; Zirrolli, J. A.; Murphy, R. C. J. Am. Soc. Mass Spectrom. 1996, 7, 129-139. 